\title{
Radiation doses in esophageal cancer
}

\author{
Susan A. Smith ${ }^{1}$, Matthew P. Fox ${ }^{2}$ \\ ${ }^{1}$ Department of Surgery, University of Louisville School of Medicine, Louisville, KY, USA; ${ }^{2}$ Department of Cardiovascular and Thoracic Surgery, \\ University of Louisville School of Medicine, Louisville, KY, USA \\ Correspondence to: Matthew P. Fox, MD. Department of Cardiovascular and Thoracic Surgery, University of Louisville School of Medicine, 201 \\ Abraham Flexner Way Suite 1200 Louisville, KY 40202, USA. Email: matthew.fox@ulp.org. \\ Provenance: This is an invited article commissioned by the Academic Editor Shuangjiang Li (Department of Thoracic Surgery and West China \\ Medical Center, West China Hospital, Sichuan University, Chengdu, China). \\ Comment on: Semenkovich, TR, Samson, PP, Hudson, JL, et al. Induction Radiation Therapy for Esophageal Cancer: Does Dose Affect Outcomes? \\ Ann Thorac Surg 2019;107:903-11.
}

Submitted Nov 26, 2019. Accepted for publication Dec 08, 2019.

doi: $10.21037 /$ jtd.2019.12.96

View this article at: http://dx.doi.org/10.21037/jtd.2019.12.96

An estimated 17,000 new cases of esophageal cancer will be diagnosed in the United States this year alone along with an estimated 16,000 deaths (1). Neoadjuvant chemotherapy with or without concurrent radiotherapy followed by surgery has emerged as a treatment of choice for locally-advanced esophageal cancer after several studies demonstrated a survival benefit of concurrent neoadjuvant chemoradiotherapy versus surgery alone (2).

The Cancer and Leukemia Group B (CALGB) 9781 Trial was the first to show improved survival in patients who received neoadjuvant cisplatin and 5 -fluorouracil with concurrent 50.4 Gy radiotherapy (3). Median survival was 4.48 versus 1.79 years in patients who underwent surgical resection alone; 5-year survival rate among the treatment arm was $39 \%$ versus $16 \%$ (3).

Dose escalation was attempted in the United States Intergroup Study 0123 (INT0123), which compared the standard radiation doses of 50.4 Gy as used in CALGB with high-dose radiation (64.4 Gy) when given concurrently with cisplatin and 5-fluorouracil (4). This study showed no significant difference in median survival, 2-year survival, or locoregional control between high-dose and standard-dose radiation, thus supporting the use of standard doses to limit local toxicity. However, none of these patients underwent surgical resection (4).

The standard dose of 50.4 Gy was primarily used until the Chemoradiotherapy for Oesophageal Cancer Followed by Surgery Study (CROSS trial), which showed improved survival in patients who received neoadjuvant carboplatin and paclitaxel with concurrent 41.4 Gy external beam radiation (5). This regimen resulted in an $\mathrm{R} 0$ resection in $92 \%$ of patients as well as median overall survival of 49.4 months compared to 24 months in the surgery alone cohort. Pathologic complete response was similar to that of CALGB at 29\% (5) versus 30\% with a dose of 50.4 Gy (3). Moreover, no difference was found in postoperative morbidity and mortality (5).

While the National Comprehensive Cancer Network (NCCN) Guidelines recommend standard preoperative doses of 41.4 to 50.4 Gy based on these studies, induction radiation doses actually used vary (6). Despite its value in establishing locoregional control while minimizing postoperative complications, few studies directly comparing preoperative radiation doses prior to this paper have been performed, so no optimal dose has been established $(2,7)$.

Semenkovich et al. reviewed the National Cancer Database (NCDB) to identify all patients with esophageal cancer who underwent neoadjuvant radiation therapy prior to esophagectomy (2). They subsequently categorized patients based on induction radiation dose as low dose (less than $40 \mathrm{~Gy}$ ), standard dose (40 to $50.4 \mathrm{~Gy}$ ), or high dose (greater than 50.4 to $70 \mathrm{~Gy}$ ) to determine differences in short and long-term outcomes after esophagectomy, including tumor response, perioperative morbidity and mortality, and overall survival. They hypothesized that increased morbidity was associated with high doses, whereas lower rates of tumor response were associated with low doses (2). 
Over the study period of 2004 to 2014, the frequency of standard-dose radiation increased from $70 \%$ to $90 \%$ whereas the frequency of low-dose radiation decreased. Analysis demonstrated higher rates of pathologic complete response (low 11.7\%; standard 16.2\%; high 21\%; P<0.001) and subsequent down-staging (low 52\%; standard 56.4\%; high $63.1 \% ; \mathrm{P}<0.001$ ) as the radiation dose increased, and treatment at an academic center was associated with both higher dose and tumor response. High-dose radiation was independently associated increased mortality at both 30 days [odds ratio (OR) 2.11; 95\% confidence interval (95\% CI), 1.47 to 3.03 ] and 90 days (OR 1.59; $95 \%$ CI, 1.21 to 2.09). However, no significant differences in prolonged hospital stay and 30-day readmission rates among the groups were demonstrated. In sub-analysis of the standard dose group, slightly higher rates of pathologic complete response were seen in those who received 50.4 versus 45 Gy $(17.3 \%$ versus $15 \% ; \mathrm{P}=0.003)$, but no difference was seen in the number of positive lymph nodes, 30-day readmission, and mortality at 30 and 90 days. Thus, this study determined that the ideal induction radiation dose to maximize tumor response without increasing perioperative morbidity and mortality is, in fact, the standard dose of 40 to 50.4 Gy. (2)

Similarly, Ising et al. reviewed the NCDB to study the influence of standard neoadjuvant radiation dose in patients undergoing esophagectomy, specifically comparing 41.4 Gy with $50 \mathrm{~Gy} / 50.4 \mathrm{~Gy}$ doses (7). The frequency of 41.4 Gy doses increased from $4.7 \%$ to $20.7 \%$ over the study period. They found that the rate of esophagectomy was greater in patients who received $41.4 \mathrm{~Gy}(87.0 \%$ versus $82.1 \% ; \mathrm{P}=0.008)$ but that postoperative length of stay, complete pathologic response, R0 resection, and 30 and 90-day mortality were not significantly different between the two groups. Interestingly, they found no difference in overall survival between 41.4 Gy and 50 Gy/50.4 Gy even in patients who ultimately did not undergo esophagectomy supporting the use of lower doses over higher doses in the neoadjuvant setting. Although analysis did not provide information regarding locoregional control, they concluded that the likelihood of undergoing esophagectomy and thus R0 resection may be increased with the use of lower radiation doses without affecting overall survival (7).

Subgroup analysis in Semenkovich et al. comparing histologic subtypes showed an association between increased dose and increased rates of complete pathologic response as well as 90-day mortality for both squamous cell carcinoma and adenocarcinoma. Interestingly, multivariate analysis demonstrated an independent association between radiation dose and 90-day mortality in the squamous cell carcinoma subgroup, whereas association was not found for adenocarcinoma (2). While the cause of this effect is unknown, this is potentially a consequence of the midthoracic location of squamous cell carcinomas (for which was not controlled in this study) and the development of catastrophic side effects such as aortoesophageal fistula or tracheoesophageal fistula. In future studies, it would be interesting to see if high-dose radiation to the distal esophagus avoids this mortality increase and is safe while still maintaining the same complete pathologic response rate.

Sufficient randomized control trial data exists demonstrating that radiation doses over 50.4 Gy do not improve morbidity or overall survival. The authors support the routine use of $50.4 \mathrm{~Gy}$ due to the rate of complete pathologic response without increase in perioperative morbidity and mortality. Their logic is reasonable though we would disagree based on the lack of increased tumor response and similar overall survival in patients who received $41.4 \mathrm{~Gy}$, even in those who did not undergo esophagectomy. Ultimately, the differences between 41.4 and 50.4 Gy may not truly be clinically significant but equipoise does exist to support a randomized controlled trial.

\section{Acknowledgments}

None.

\section{Footnote}

Conflicts of Interest: The authors have no conflicts of interest to declare.

Ethical Statement: The authors are accountable for all aspects of the work in ensuring that questions related to the accuracy or integrity of any part of the work are appropriately investigated and resolved.

\section{References}

1. Siegel RL, Miller KD, Jemal A. Cancer statistics, 2019. CA Cancer J Clin 2019;69:7-34.

2. Semenkovich TR, Samson PP, Hudson JL, et al. Induction Radiation Therapy for Esophageal Cancer: Does Dose Affect Outcomes? Ann Thorac Surg 2019;107:903-11.

3. Tepper J, Krasna MJ, Niedzwiecki D, et al. Phase III 
trial of trimodality therapy with cisplatin, fluorouracil, radiotherapy, and surgery compared with surgery alone for esophageal cancer: CALGB 9781. J Clin Oncol 2008;26:1086-92.

4. Minsky BD, Pajak TF, Ginsberg RJ, et al. INT 0123 (Radiation Therapy Oncology Group 94-05) phase III trial of combined-modality therapy for esophageal cancer: high-dose versus standard-dose radiation therapy. J Clin Oncol 2002;20:1167-74.

5. van Hagen $\mathrm{P}$, Hulshof MC, van Lanschot JJ, et al.

Cite this article as: Smith SA, Fox MP. Radiation doses in esophageal cancer. J Thorac Dis 2019;11(12):5688-5690. doi: 10.21037/jtd.2019.12.96
Preoperative chemoradiotherapy for esophageal or junctional cancer. N Engl J Med 2012;366:2074-84.

6. Ajani JA, D'Amico TA, Bentrem DJ, et al. Esophageal and Esophagogastric Junction Cancers, Version 2.2019, NCCN Clinical Practice Guidelines in Oncology. J Natl Compr Canc Netw 2019;17:855-83.

7. Ising MS, Marino K, Trivedi JR, et al. Influence of Neoadjuvant Radiation Dose on Patients Undergoing Esophagectomy and Survival in Locally Advanced Esophageal Cancer. J Gastrointest Surg 2019;23:670-8. 